\title{
Particle-Hole Duality, Emergent Fermi Liquids, and Fractional Chern Insulators in Moiré Flatbands
}

\author{
Ahmed Abouelkomsan $\oplus^{1}{ }^{2}$ Zhao Liu $\odot,{ }^{2, *}$ and Emil J. Bergholtz $\oplus^{1, \dagger}$ \\ ${ }^{1}$ Department of Physics, Stockholm University, AlbaNova University Center, 10691 Stockholm, Sweden \\ ${ }^{2}$ Zhejiang Institute of Modern Physics, Zhejiang University, Hangzhou 310027, China
}

(Received 14 December 2019; accepted 12 February 2020; published 11 March 2020)

\begin{abstract}
Moiré flatbands, occurring, e.g., in twisted bilayer graphene at magic angles, have attracted ample interest due to their high degree of experimental tunability and the intriguing possibility of generating novel strongly interacting phases. Here we consider the core problem of Coulomb interactions within fractionally filled spin and valley polarized Moiré flatbands and demonstrate that the dual description in terms of holes, which acquire a nontrivial hole dispersion, provides key physical intuition and enables the use of standard perturbative techniques for this strongly correlated problem. In experimentally relevant examples such as $\mathrm{ABC}$ stacked trilayer and twisted bilayer graphene aligned with boron nitride, it leads to emergent interaction-driven Fermi liquid states at electronic filling fractions down to around $1 / 3$ and $2 / 3$, respectively. At even lower filling fractions, the electron density still faithfully tracks the single-hole dispersion while exhibiting distinct non-Fermi liquid behavior. Most saliently, we provide microscopic evidence that high temperature fractional Chern insulators can form in twisted bilayer graphene aligned with hexagonal boron nitride.
\end{abstract}

DOI: $10.1103 /$ PhysRevLett.124.106803

Introduction.-Moiré superlattice systems have attracted enormous interest following the discovery of unconventional superconductivity and correlated states in twisted bilayer graphene near the magic angle [1-6]. Owing to experimental advances in manufacturing van der Waals heterostructures [7], there have been an increasing number of experiments investigating a plethora of novel strongly correlated two-dimensional systems with Moiré patterns. A salient example is bilayer graphene at a magic twist angle [8-12] aligned with hexagonal boron nitride which gaps out the protected Dirac points of the graphene and the flatbands around charge neutrality acquire nonzero Chern numbers [13-16]. Here, recent experiments show both emergent ferromagnetism [17] and a quantized anomalous Hall effect [18] at different integer fillings suggesting that the underlying state is a Chern insulator.

A related platform for studying correlated physics is the Moiré superlattice formed when trilayer graphene is aligned with boron nitride. Unlike magic angle twisted bilayer graphene, the bandwidth and the topology of the minibands can be controlled by the strength of the applied displacement field resulting in gate-tunable Mott insulating and superconducting states $[19,20]$. Also in this case, a

Published by the American Physical Society under the terms of the Creative Commons Attribution 4.0 International license. Further distribution of this work must maintain attribution to the author(s) and the published article's title, journal citation, and DOI. Funded by Bibsam. correlated ferromagnetic Chern insulator has been reported at a filling corresponding to one hole per Moiré unit cell [21].

While a tremendous amount of attention has been paid to the aforementioned Moiré flatband systems, the detailed microscopic understanding of the crucial impact of Coulomb interactions has essentially been limited to investigating the spin and valley polarization at integer fillings of Moiré flatbands [22].

In sharp contrast, in this Letter, we provide a detailed microscopic study of Coulomb interactions within a fractionally filled spin-polarized Moiré flatband near one valley relevant for the experimental situation at finite doping. Motivated by the aforementioned recent experiments we focus on two particularly intriguing models. The first being $\mathrm{ABC}$ stacked trilayer graphene aligned with boron nitride which we refer to as TLG-hBN [23-27]. Here we take our fractionally filled band to be the valence band with Chern number $C=3$ and the valence band with Chern number $C=0$ obtained by switching the sign of the applied voltage. The second model describes twisted bilayer graphene aligned with boron nitride [28] which we refer to as TBG-hBN. The active band is taken to be the valence band with Chern number $C=1$.

Based on the glaring analogy with Landau levels, it has been suggested that exotic correlated states such as fractional Chern insulators (FCIs) may quite generically form in $C \neq 0$ Moiré flatbands [24]. Remarkably, however, we find that a large portion of the phase diagram at fractional filling in each of these bands is dominated by particle-hole 
symmetry breaking terms that are absent in Landau levels: rewriting the interaction Hamiltonian in terms of holes yields a dual formulation whereby the strongly interacting electron problem is converted into an effectively weakly interacting hole problem that is amenable to standard perturbative approaches. This results in a new paradigm for Fermi liquid states, with telltale experimental signatures, emerging from projected interactions alone. Moreover, our microscopic calculations also indicate instabilities at low electronic filling where the interactions, notwithstanding the paramount impact of the hole dispersion, lead to genuinely strongly correlated states including a high-temperature fractional Chern insulator.

Setup.-Motivated by the recent discoveries [17-19,21] along with theoretical predictions $[22,29,30]$ of spin and valley polarized insulators at integer fillings in various Moiré bands, we consider electrons with polarized spin and valley degrees of freedom interacting via the screened Coulomb potential

$$
H_{a, \sigma}=\frac{1}{2} \sum_{\mathbf{q} \in R^{2}} V(\mathbf{q}): \rho_{a, \sigma}(\mathbf{q}) \rho_{a, \sigma}(-\mathbf{q}):
$$

where $\rho_{a \sigma}(\mathbf{q})$ is the density operator in valley $a$ with spin $\sigma$. We choose the Yukawa potential $V(\mathbf{q})=$ $\left(e^{2} / 4 \pi \epsilon S\right)\left(2 \pi / \sqrt{|\mathbf{q}|^{2}+\kappa^{2}}\right)$ to describe the screening of the Coulomb interaction, where $e$ is the electron charge, $\epsilon$ is the dielectric constant of the material, $S$ is the area of the Moiré superlattice, and $\kappa$ measures the screening strength [31]. While the full interaction Hamiltonian has $U(2) \times U(2)$ symmetry including separate charge and spin conservation within each valley, we in Eq. (1) neglect weaker terms like intervalley scattering and Hund's coupling which would break this symmetry.

When the electrons partially fill a Moiré flatband in valley $a$ with spin $\sigma$ while all other bands are either empty or completely filled, it is fair to project the interaction (1) to the active flatband and neglect the band dispersion, so that the underlying physics driven by strong interactions is emphasized and disentangled from the quantitatively small effect of a remnant band dispersion. The projected Hamiltonian has the form

$H_{a, \sigma}^{\mathrm{proj}}=\sum_{\left\{\mathbf{k}_{\mathbf{i}}\right\}} V_{\mathbf{k}_{1} \mathbf{k}_{2} \mathbf{k}_{3} \mathbf{k}_{4}}^{a} c_{a, \sigma}^{\dagger}\left(\mathbf{k}_{1}\right) c_{a, \sigma}^{\dagger}\left(\mathbf{k}_{2}\right) c_{a, \sigma}\left(\mathbf{k}_{3}\right) c_{a, \sigma}\left(\mathbf{k}_{4}\right)$,

where $c_{a, \sigma}^{\dagger}(\mathbf{k})\left(c_{a, \sigma}(\mathbf{k})\right)$ creates (annihilates) an electron with momentum $\mathbf{k}$ and spin $\sigma$ in the partially filled flatband in valley $a$, and all $\mathbf{k}_{i}$ 's are in the Moire Brillouin zone (MBZ). The matrix element $V_{\mathbf{k}_{1} \mathbf{k}_{2} \mathbf{k}_{3} \mathbf{k}_{4}}^{a, \sigma}$ can be derived based on the effective model of the pertinent Moiré superlattice as detailed in the Supplemental Material [31]. In what follows, we impose periodic boundary conditions on finite Moiré superlattices and use extensive exact diagonalization to study the low-energy properties of the Hamiltonian (2) at various filling factors $\nu=N /\left(N_{1} N_{2}\right)$, where $N$ is the number of spin- $\sigma$ electrons in the active band of valley $a$ and $N_{1}$ and $N_{2}$ are the number of unit cells in the two basic directions $\mathbf{a}_{1}$ and $\mathbf{a}_{2}$ of the Moiré superlattice. We choose $\frac{1}{2}\left(e^{2} / 4 \pi \epsilon a_{M}\right)$ as the energy unit, where $a_{M}$ is the lattice constant of the Moiré superlattice. Unless otherwise stated we set the Coulomb screening strength as $\kappa=1 / a_{M}$.

Particle-hole duality and emergent Fermi liquids.From now on, we drop the spin and valley indices for simplicity. Upon a particle-hole transformation $c(\mathbf{k}) \rightarrow d^{\dagger}(\mathbf{k})$, the interaction Hamiltonian (2) becomes

$$
\begin{aligned}
H^{\text {proj }} \rightarrow & \sum_{\mathbf{k} \in \mathrm{MBZ}} E_{h}(\mathbf{k}) d^{\dagger}(\mathbf{k}) d(\mathbf{k}) \\
& +\sum_{\left\{\mathbf{k}_{\mathbf{i}}\right\} \in \mathrm{MBZ}} V_{\mathbf{k}_{1} \mathbf{k}_{2} \mathbf{k}_{3} \mathbf{k}_{4}}^{*} d^{\dagger}\left(\mathbf{k}_{1}\right) d^{\dagger}\left(\mathbf{k}_{2}\right) d\left(\mathbf{k}_{3}\right) d\left(\mathbf{k}_{4}\right)
\end{aligned}
$$

with

$E_{h}(\mathbf{k})=\sum_{\mathbf{k}^{\prime} \in \mathrm{MBZ}}\left(V_{\mathbf{k}^{\prime} \mathbf{k} \mathbf{k}^{\prime} \mathbf{k}}+V_{\mathbf{k} \mathbf{k}^{\prime} \mathbf{k} \mathbf{k}^{\prime}}-V_{\mathbf{k k}^{\prime} \mathbf{k}^{\prime} \mathbf{k}}-V_{\mathbf{k}^{\prime} \mathbf{k} \mathbf{k} \mathbf{k}^{\prime}}\right)$

As a result, the dual Hamiltonian expressed in terms of hole operators acquires an interaction-induced single-hole dispersion which is nonconstant for generic projected interactions, as shown in Fig. 1. For some simple toy
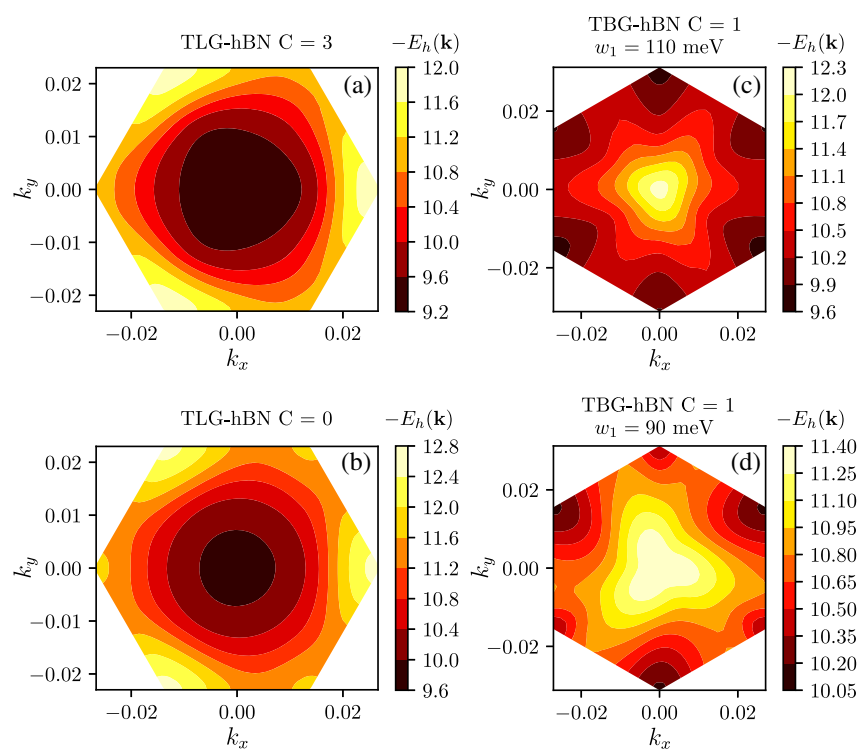

FIG. 1. Contour plots of the single-hole dispersion $-E_{h}(\mathbf{k})$ in the Moiré Brillouin zone for (a) the TLG-hBN $C=3$ valence band, (b) TLG-hBN $C=0$ valence band, (c) TBG-hBN $C=1$ valence band with $w_{1}=110 \mathrm{meV}$, and (d) TBG-hBN $C=1$ valence band with $w_{1}=90 \mathrm{meV} . w_{1}$ is the off-diagonal tunneling strength in TBG [31]. The inverse screening length is $\kappa=1 / a_{M}$. 
flatband models designed for finding fractional Chern insulators in systems with a small unit cell, it has been observed that such a dispersion dominates over the holehole interaction at large electron fillings $\nu \gtrsim 4 / 5$, thus destabilizing FCIs and resulting in Fermi liquidlike states $[32,33]$.

Remarkably, we find that the effect of the particle-hole symmetry breaking terms [Eq. (4)] is much more pronounced and dictates the physics also far away from the dilute hole limit in the experimentally relevant Moiré flatband systems. This is manifest in Figs. 2(a)-2(d) that display the ground-state electron density $\langle n(\mathbf{k})\rangle=$ $\left\langle c^{\dagger}(\mathbf{k}) c(\mathbf{k})\right\rangle$ parametrized by the effective single-hole energy $-E_{h}(\mathbf{k})$ at various electron fillings $1 / 7 \leq \nu \leq$ 9/10 in TLG-hBN and TBG-hBN. In all cases that we study, the electron density is quite generally strongly dependent on-and essentially a monotonic function of the effective single-hole energy. This correspondence becomes even more striking when Hartree-Fock corrections to the single-hole energy are accounted for with a renormalized hole dispersion [31]

$$
\begin{aligned}
\tilde{E}_{h}(\mathbf{k})= & \frac{1}{\nu} \sum_{\mathbf{k}^{\prime}}\left\langle n\left(\mathbf{k}^{\prime}\right)\right\rangle \\
& \times\left(V_{\mathbf{k k}^{\prime} \mathbf{k} \mathbf{k}^{\prime}}+V_{\mathbf{k}^{\prime} \mathbf{k} \mathbf{k}^{\prime} \mathbf{k}}-V_{\mathbf{k k}^{\prime} \mathbf{k}^{\prime} \mathbf{k}}-V_{\mathbf{k}^{\prime} \mathbf{k} \mathbf{k} \mathbf{k}^{\prime}}\right),
\end{aligned}
$$

which leads to a slightly modified parametrization of $\langle n(\mathbf{k})\rangle$ as shown in Figs. 2(e)-2(h).

Saliently, we find clearly resolved emergent Fermi liquid behavior signaled by a sharp step in the electronic occupation numbers at electron fillings extending all the way down to $\nu=1 / 3$ for the valence bands of TLG-hBN. While this is already quite clear from the bare single-hole dispersion [Figs. 2(a)-2(b)], the renormalized hole energy removes occasional oscillations of the density close to the emergent Fermi surface, making it remarkably well defined despite the relatively small systems available in our exact diagonalization study [Figs. 2(e)-2(f)]. The emergent Fermi surfacelike structure naturally becomes increasingly sharp at increasing $\nu$, corroborating that the ground states at those fillings are indeed weakly interacting Fermi liquids parametrized by the hole dispersion. Although the Fermi surface feature starts to dissolve below $\nu=1 / 3$, the strong correlation between electron density and hole dispersion persists even at fillings as low as $\nu=1 / 7$ [Figs. 2(a)-2(b) and 2(e)-2(f)], suggesting that the hole dispersion still plays an important role in the low-energy physics at very low electron fillings and that perturbative approaches can be applied to analyze at least some of the resulting instabilities.

Albeit still significant, the correspondence between the electron density and the emergent hole dispersion is less pronounced for the TBG-hBN valence band with $C=1$. In this case, we generally observe more uniform electron densities without clear Fermi surface features at small electron fillings and emergent Fermi surfaces only at comparably larger $\nu$ [Figs. 2(c) and 2(g)]. At slightly weaker tunneling between two graphene layers this tendency is further enhanced [Figs. 2(d) and 2(h)] as can be rationalized from the reduced bandwidth of the single-hole dispersion [compare Fig. 1(d) with Figs. 1(a)-1(c)]. TBG$\mathrm{hBN}$ thus stands out as a particularly promising host for genuinely strongly correlated topological states like fractional Chern insulators.
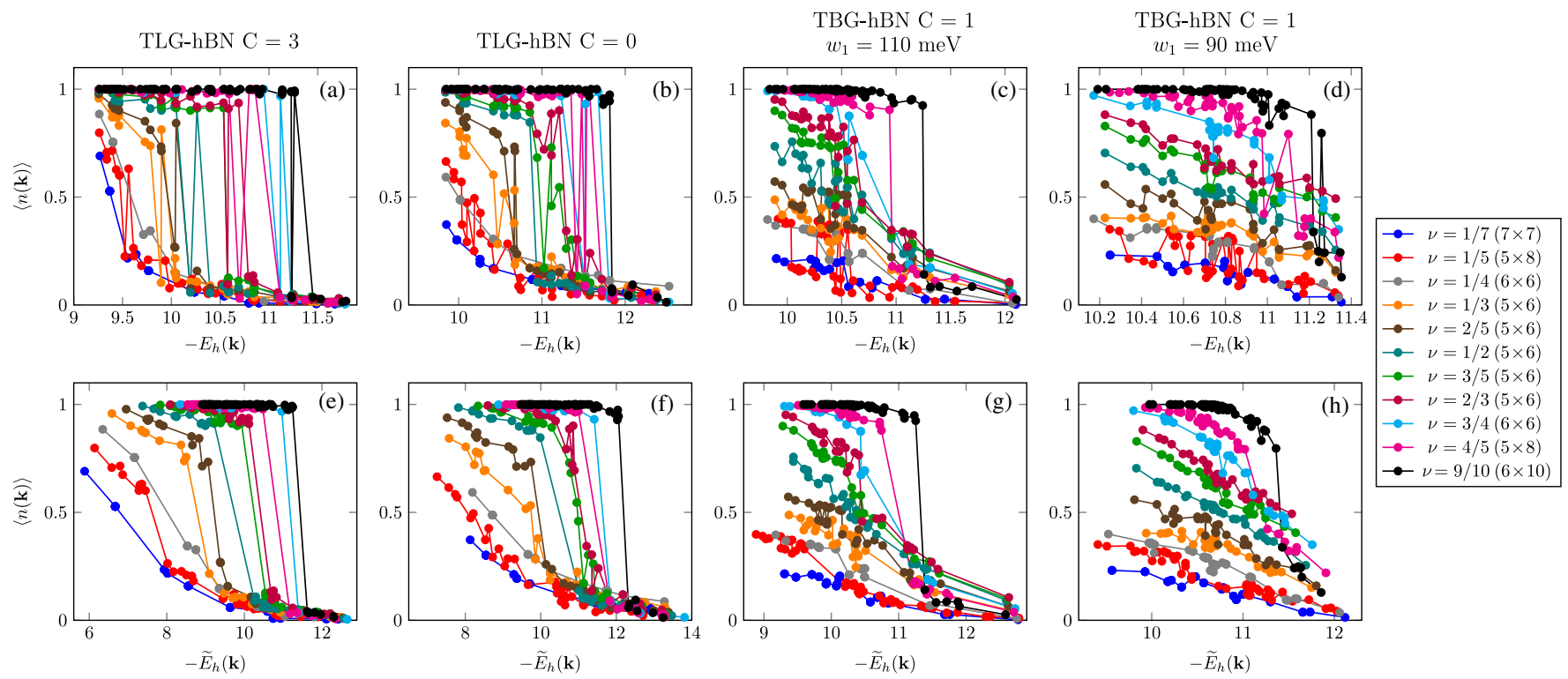

FIG. 2. The ground-state electron density $\langle n(\mathbf{k})\rangle$ versus [(a)-(d)] the original hole dispersion $-E_{h}(\mathbf{k})$ and [(e)-(h)] the renormalized hole dispersion $-\tilde{E}_{h}(\mathbf{k})$ at various electron fillings $\nu$. Note the different off-diagonal tunneling strength $w_{1}$ between two graphene layers [31] in [(c),(g)] and [(d),(h)]. The parentheses next to $\nu$ denote the system size $N_{1} \times N_{2}$. The inverse screening length is $\kappa=1 / a_{M}$. 
(a)

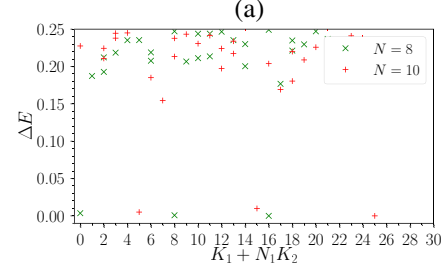

(b)

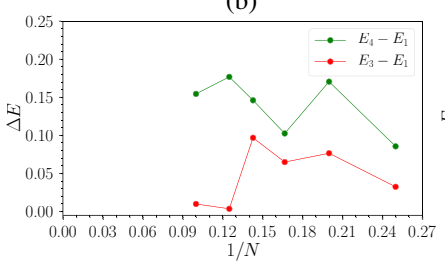

(c)

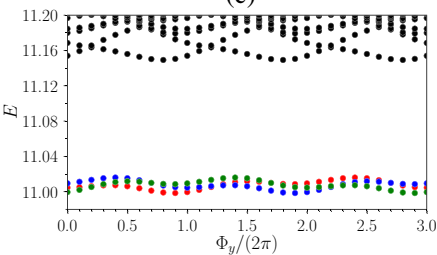

(d)

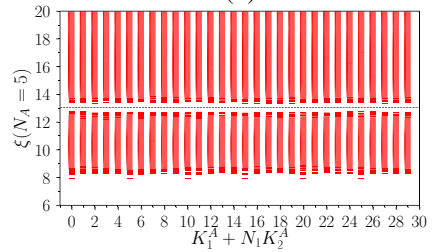

FIG. 3. Evidence of $\nu=1 / 3$ FCIs in the $C=1$ band of TBG-hBN. Here we choose $w_{1}=90$ meV [31] and the inverse screening length is $\kappa=1 / a_{M}$. (a) The low-lying energy spectrum for $N=8, N_{1} \times N_{2}=4 \times 6$ and $N=10, N_{1} \times N_{2}=5 \times 6$. (b) The finite-size scaling of the energy gap (green) and the ground-state splitting (red) for $N=4-10$. We define the energy gap and the ground-state splitting as $E_{4}-E_{1}$ and $E_{3}-E_{1}$, respectively, where $E_{i}$ is the $i$ th energy level in ascending order. (c) The spectral flow for $N=10$, $N_{1} \times N_{2}=5 \times 6$, where $\Phi_{y}$ is the magnetic flux insertion in the $\mathbf{a}_{2}$ direction. (d) The particle entanglement spectrum for $N=10$, $N_{1} \times N_{2}=5 \times 6$, with 23256 levels below the entanglement gap (the dashed line).

Fractional Chern insulators.-Now we address the possibility of FCIs stabilized by the screened Coulomb interaction in the $C=1$ band of TBG-hBN. We consider electronic band filling $\nu=1 / 3$ where one may on general grounds anticipate that FCIs may exist and focus the case with off-diagonal tunneling strength $w_{1}=90 \mathrm{meV}$ encouraged by the relatively weak single-hole dispersion [Fig. 1(d)] and comparably featureless electron density profile [Figs. 2(d) and 2(h)] at this parameter value. As detailed below and in Fig. 3 we indeed find compelling numerical evidence of stable high-temperature $\nu=1 / 3$ FCIs.

First we examine the energetics of the Hamiltonian (2). For various system sizes that we study, there are three approximately degenerate states at the bottom of the energy spectrum, whose total momentum $\left(K_{1}, K_{2}\right)$ are consistent with the prediction of Haldane statistics [34-36] for the $\nu=$ $1 / 3$ FCIs. These three ground states are separated from excited states by a clear energy gap, which becomes larger than the ground-state splitting as the system size grows [Fig. 3(a)]. A finite-size scaling of the energy gap demonstrates that it is very likely to survive in the thermodynamic limit [Fig. 3(b)]. Moreover, the threefold ground-state degeneracy persists under magnetic flux insertion through the handles of the toroidal system [Fig. 3(c)], which confirms the robustness of topological degeneracy.

To further corroborate that the ground states are topologically nontrivial, we have investigated the particle entanglement spectrum (PES), which contains information of the excitation structure of the system and can be used to distinguish FCIs from competing possibilities such as charge density waves $[34,37,38]$. The result is displayed in Fig. 3(d), where we divide the whole system into $N_{A}$ and $N-N_{A}$ electrons and label each PES level by the total momentum $\left(K_{1}^{A}, K_{2}^{A}\right)$ of those $N_{A}$ electrons. We find a clear entanglement gap separating the low-lying PES levels from higher ones, and the number of levels below the gap exactly matches the pertinent counting of quasihole excitations in the $\nu=1 / 3$ Abelian FCI states [34-36].

We have also considered the situation with larger interlayer tunneling in TBG-hBN. We increase $w_{1}$ to $110 \mathrm{meV}$, and again we observe clear signatures of FCI states albeit only at somewhat stronger screening $\kappa=6 / a_{M}$ [31]. Moreover, we find that projecting the interaction to the other $C=-1$ band of TBG-hBN gives very similar results to Fig. 3 [31], indicating that FCI states can exist in both topological bands of this model.

Discussion.-We have shown that the dual description of Coulomb interactions in partially filled Moiré flatbands in terms of holes provides remarkable insights about this strongly correlated problem, turning it into an effectively weakly interacting problem in a large portion of the phase diagram. In particular, this identifies ABC stacked trilayer and twisted bilayer graphene aligned with boron nitride as realistic hosts of a new paradigm for emergent Fermi liquids deriving from essentially pure interaction effects at appropriate doping. This perspective opens up a number of intriguing experimental prospects as well as providing a key quantity-the single-hole dispersion, $E_{h}(\mathbf{k})$-with a bandwidth estimate that is typically significantly larger than the flatband bandwidth [31]. It enables the use of standard many-body techniques which are typically not applicable to flatband systems due to the apparent lack of a small parameter. In particular, our simple Hartree-Fock calculations for the holes corroborate the generic applicability of the interaction driven Fermi liquid phenomenology.

The exotic interaction induced Fermi surfaces come with telltale experimental signatures in terms of quantum oscillations (cf. Fig. 1). In this context it is worth noting that there are plenty of experimentally tunable knobs that can alter the shape of these Fermi surfaces-and eventually cause their breakdown. While some instabilities invite future research, such as those leading to a convex density profile at low filling fractions in the trilayer graphene setup (cf. Fig. 2), we have investigated in detail the case when the Fermi surface is smeared in the most extreme sense with an essentially featureless density profile only weakly linearly correlated with the hole energy. Here we provided numerical evidence that fractional Chern insulators may form in TBG-hBN at electronic band filling $\nu=1 / 3$. It is quite remarkable that such state is stabilized in a realistic model systems with long-range Coulomb interactions (screening 
length $\sim a_{M} \sim 60$ graphene lattice constants [39]) in stark contrast to previously studied toy models which generically feature strictly short-range interactions. Here, again, the hole dispersion provided key intuition: only for models with a small hole bandwidth there is a chance of realizing FCIs. This in turn allows us to develop an intuition for which externally tunable parameters that may be alter the phase diagram to achieve the desired many-body states. In particular, we have found either changing the screening length or the interlayer tunneling, which are tunable through pressure and substrate engineering, respectively, can effectively (de)stabilize FCIs [31].

Another remarkable feature of the interacting Moiré flatbands is the large energy scale associated with the projected Coulomb interaction; a rough estimate of the stability of the FCIs based on the finite-size gap calculations (cf. Fig. 3) suggests that they may persist all the way up to $\sim 10 \mathrm{~K}$. This thus identifies twisted bilayer graphene aligned with boron nitride as a particularly promising platform for finding observing the first bona fide FCIsat elevated temperatures and in absence of an external magnetic field. Moreover, our numerical evidence of $\nu=$ $1 / 3$ FCIs in TBG-hBN, invites a systematic search for fractionalized topological states at other fillings and in Moiré flatbands derived from other materials.

Finally, we stress that we have thus far only considered a single fractionally filled spin-valley sector. While this is a very reasonable assumption for spatially homogenous phases, the multicomponent nature of the problem and effects of symmetry breaking are expected to further enrich the phenomenology of interacting Moiré flatbands. Also in this context we suggest that the dual formulations in terms of holes will likely provide key physical insights.

We would like to thank Johan Carlström and Roderich Moessner for useful discussions. A. A. and E. J. B. are supported by the Swedish Research Council (VR) and the Wallenberg Academy Fellows program of the Knut and Alice Wallenberg Foundation. Z. L. is supported by the National Natural Science Foundation of China through Grant No. 11974014.

*zhaol@zju.edu.cn

†emil.bergholtz@fysik.su.se

[1] Y. Cao, V. Fatemi, S. Fang, K. Watanabe, T. Taniguchi, E. Kaxiras, and P. Jarillo-Herrero, Unconventional superconductivity in magic-angle graphene superlattices, Nature (London) 556, 43 (2018).

[2] Y. Cao, V. Fatemi, A. Demir, S. Fang, S. L. Tomarken, J. Y. Luo, J. D. Sanchez-Yamagishi, K. Watanabe, T. Taniguchi, E. Kaxiras et al., Correlated insulator behaviour at half-filling in magic-angle graphene superlattices, Nature (London) $\mathbf{5 5 6}$, 80 (2018).

[3] M. Yankowitz, S. Chen, H. Polshyn, Y. Zhang, K. Watanabe, T. Taniguchi, D. Graf, A. F. Young, and
C. R. Dean, Tuning superconductivity in twisted bilayer graphene, Science 363, 1059 (2019).

[4] X. Lu, P. Stepanov, W. Yang, M. Xie, M. A. Aamir, I. Das, C. Urgell, K. Watanabe, T. Taniguchi, G. Zhang, A. Bachtold, A. H. MacDonald, and D. K. Efetov, Superconductors, orbital magnets and correlated states in magic-angle bilayer graphene, Nature (London) 574, 653 (2019).

[5] Y. Xie, B. Lian, B. Jäck, X. Liu, C. Chiu, K. Watanabe. T. Taniguchi, B. A. Bernevig, and A. Yazdani, Spectroscopic signatures of many-body correlations in magic-angle twisted bilayer graphene, Nature (London) 572, 101 (2019).

[6] H. C. Po, L. Zou, A. Vishwanath, and T. Senthil, Origin of Mott Insulating Behavior and Superconductivity in Twisted Bilayer Graphene, Phys. Rev. X 8, 031089 (2018).

[7] A. Geim and I. Grigorieva, Van der Waals heterostructures, Nature (London) 499, 419 (2013).

[8] R. Bistritzer and A. H. MacDonald, Moiré bands in twisted double-layer graphene, Proc. Natl. Acad. Sci. U.S.A. 108, 12233 (2011).

[9] J. M. B. Lopes dos Santos, N. M. R. Peres, and A. H. Castro Neto, Continuum model of the twisted graphene bilayer, Phys. Rev. B 86, 155449 (2012).

[10] J. M. B. Lopes dos Santos, N. M. R. Peres, and A. H. Castro Neto, Graphene Bilayer with a Twist: Electronic Structure, Phys. Rev. Lett. 99, 256802 (2007).

[11] G. Tarnopolsky, A. J. Kruchkov, and A. Vishwanath, Origin of Magic Angles in Twisted Bilayer Graphene, Phys. Rev. Lett. 122, 106405 (2019).

[12] M. Koshino, N. F. Q. Yuan, T. Koretsune, M. Ochi, K. Kuroki, and L. Fu, Maximally Localized Wannier Orbitals and the Extended Hubbard Model for Twisted Bilayer Graphene, Phys. Rev. X 8, 031087 (2018).

[13] J. Jung, A. M. DaSilva, A. H. MacDonald, and S. Adam, Origin of band gaps in graphene on hexagonal boron nitride, Nat. Commun. 6, 6308 (2015).

[14] P. San-Jose, A. Gutiérrez-Rubio, M. Sturla, and F. Guinea, Spontaneous strains and gap in graphene on boron nitride, Phys. Rev. B 90, 075428 (2014).

[15] F. Amet, J. R. Williams, K. Watanabe, T. Taniguchi, and D. Goldhaber-Gordon, Insulating Behavior at the Neutrality Point in Single-Layer Graphene, Phys. Rev. Lett. 110, 216601 (2013).

[16] B. Hunt, J. D. Sanchez-Yamagishi, A. F. Young, M. Yankowitz, B. J. LeRoy, K. Watanabe, T. Taniguchi, P. Moon, M. Koshino, P. Jarillo- Herrero, and R. C. Ashoori, Massive Dirac fermions and Hofstadter butterfly in a van der Waals heterostructure, Science 340, 1427 (2013).

[17] A. L. Sharpe, E. J. Fox, A. W. Barnard, J. Finney, K. Watanabe, T. Taniguchi, M. A. Kastner, and D. GoldhaberGordon, Emergent ferromagnetism near three-quarters filling in twisted bilayer graphene, Science 365, 605 (2019).

[18] M. Serlin, C. L. Tschirhart, H. Polshyn, Y. Zhang, J. Zhu, K. Watanabe, T. Taniguchi, L. Balents, and A. F. Young, Intrinsic quantized anomalous Hall effect in a Moiré heterostructure, arXiv:1907.00261.

[19] G. Chen, L. Jiang, S. Wu, B. Lyu, H. Li, B. L Chittari, K. Watanabe, T. Taniguchi, Z. Shi, J. Jung et al., Evidence of a gate-tunable Mott insulator in a trilayer graphene Moiré superlattice, Nat. Phys. 15, 237 (2019). 
[20] G. Chen, A. L. Sharpe, P. Gallagher, I. T. Rosen, E. J. Fox, L. Jiang, B. Lyu, H. Li, K. Watanabe, T. Taniguchi, J. Jung, Z. Shi, D. Goldhaber-Gordon, Y. Zhang, and F. Wang, Signatures of tunable superconductivity in a trilayer graphene Moiré superlattice, Nature (London) 572, 215 (2019).

[21] G. Chen, A. L. Sharpe, E. J. Fox, Y. Zhang, S. Wang, L. Jiang, B. Lyu, H. Li, K. Watanabe, T. Taniguchi et al., Tunable correlated Chern insulator and ferromagnetism in trilayer graphene/boron nitride Moiré superlattice, arXiv: 1905.06535.

[22] C. Repellin, Z. Dong, Y. H. Zhang, and T. Senthil, Ferromagnetism in narrow bands of Moiré superlattices, arXiv: 1907.11723.

[23] B. L. Chittari, G. Chen, Y. Zhang, F. Wang, and J. Jung, Gate-Tunable Topological Flat Bands in Trilayer Graphene Boron-Nitride Moiré Superlattices, Phys. Rev. Lett. 122, 016401 (2019).

[24] Y. H. Zhang, D. Mao, Y. Cao, P. Jarillo-Herrero, and T. Senthil, Nearly flat Chern bands in Moiré superlattices, Phys. Rev. B 99, 075127 (2019).

[25] Y.H. Zhang and T. Senthil, Bridging Hubbard model physics and quantum Hall physics in trilayer graphene/ h-BN Moiré superlattice, Phys. Rev. B 99, 205150 (2019).

[26] C. Schrade and L. Fu, Spin-valley density wave in Moiré materials, Phys. Rev. B 100, 035413 (2019).

[27] F. Zhang, B. Sahu, H. Min, and A. H. MacDonald, Band structure of $a b c$-stacked graphene trilayers, Phys. Rev. B 82, 035409 (2010).

[28] Y. H. Zhang, D. Mao, and T. Senthil, Twisted bilayer graphene aligned with hexagonal boron nitride: Anomalous Hall effect and a lattice model, Phys. Rev. Research 1, 033126 (2019).

[29] N. Bultinck, S. Chatterjee, and M. P. Zaletel, Anomalous Hall ferromagnetism in twisted bilayer graphene, arXiv: 1901.08110.
[30] T. M. R. Wolf, J. L. Lado, G. Blatter, and O. Zilberberg, Electrically Tunable Flat Bands and Magnetism in Twisted Bilayer Graphene, Phys. Rev. Lett. 123, 096802 (2019).

[31] See Supplemental Material at http://link.aps.org/ supplemental/10.1103/PhysRevLett.124.106803 for technical details of the single-particle Hamiltonians of TLG-hBN and TBG-hBN, the projected interaction, the HartreeFock corrections to the single-hole energy, comparing the bandwidth of the single-hole dispersion with the flat valence band dispersion, and further numerical data about fractional Chern insulators.

[32] A. M. Läuchli, Z. Liu, E. J. Bergholtz, and R. Moessner, Hierarchy of Fractional Chern Insulators and Competing Compressible States, Phys. Rev. Lett. 111, 126802 (2013).

[33] E. J. Bergholtz and Z. Liu, Topological flat band models and fractional Chern insulators, Int. J. Mod. Phys. B 27, 1330017 (2013).

[34] N. Regnault and B. A. Bernevig, Fractional Chern Insulator, Phys. Rev. X 1, 021014 (2011).

[35] B. A. Bernevig and N. Regnault, Emergent many-body translational symmetries of Abelian and non-Abelian fractionally filled topological insulators, Phys. Rev. B 85, 075128 (2012).

[36] Y.-L. Wu, N. Regnault, and B. A. Bernevig, Haldane statistics for fractional Chern insulators with an arbitrary Chern number, Phys. Rev. B 89, 155113 (2014).

[37] H. Li and F. D. M. Haldane, Entanglement Spectrum as a Generalization of Entanglement Entropy: Identification of Topological Order in Non-Abelian Fractional Quantum Hall Effect States, Phys. Rev. Lett. 101, 010504 (2008).

[38] A. Sterdyniak, N. Regnault, and B. A. Bernevig, Extracting Excitations from Model State Entanglement, Phys. Rev. Lett. 106, 100405 (2011).

[39] J. Jung, A. Raoux, Z. Qiao, and A. H. MacDonald, $A b$ initio theory of Moire superlattice bands in layered two-dimensional materials, Phys. Rev. B 89, 205414 (2014). 